\title{
Evaluation of Physico-Chemical, Thermal and Mechanical Properties of Sintered Graphite and Mesophase Formulations
}

\author{
Deepak Kumar $\mathbf{G}^{1}$ and Singh Arora SP2* \\ ${ }^{1}$ Department of Chemistry, Indian Institute of Space Science and Technology (IIST), Trivandrum, Kerala, India \\ ${ }^{2}$ Scientist/Engineer-SD, Indian Space Research Organisation (ISRO), Govt. of India, Tamil Nadu, India
}

\begin{abstract}
Carbon/graphite materials are widely applied to the areas of seal and friction materials. These applications exploit the exceptional properties of carbon/graphite, such as its excellent mechanical behavior at high temperature and low reactivity. However, Owing to high open porosity, and low strength conventional carbon/graphite materials prepared using pulverized coke as filler and pitch as binder fall across enormous challenge. Therefore, it is necessary to reduce the open porosity and increase in the strength along with thermal conductivity. Using natural graphite as filler instead of coke to prepare carbon blocks can avoid high-temperature graphitization and repeated dipping owing to the high degree of graphitization and high thermal stability of natural graphite; thus it can simplify the working process, save energy and reduce cost. Carbonaceous mesophase has inherent properties, such as a high softening point, high fluidity, high carbon yield, and graphitizability, which make it suitable for the fabrication of polygranular carbon materials. Furthermore, it can be used as a binder. The sintering was performed in a static furnace with argon atmosphere and compared with the same compound sintered in passage furnace with hydrogen and nitrogen atmosphere. The analysis of the properties of the tested material was performed with the aid of metallography using a scanning electron microscope, which verified the particle size distribution, chemical elements and pores present. However, for the graphite powder and zinc stearate, present in smaller percentages were disregarded its influence on the physical properties of the compound generated. Compressibility and compaction are parameters that indicate and describe the behavior of metal powders as they are compressed. The ability of a powder densification is related to compressibility. Already compaction is defined as the stability of the structure of the pressed compacted to a certain working pressure. Therefore, the natural graphite as filler and mesophase pitch as binder can be able to produce high density graphite blocks. The main aim of this work is to produce isotropic graphite material and evaluation of its properties such as high density, high thermal conductivity, high electrical resistivity, low coefficient of thermal expansion, and high compressive strength from natural graphite powder mesophase pitch.
\end{abstract}

Keywords: Natural graphite; Mesophase pitch; Density; Thermal conductivity; Electrical resistivity; Coefficient of thermal expansion; Compressive strength

\section{Introduction}

High-density artefacts of carbon and graphite have been widely applied in various fields because of their excellent properties. They are now recognized to be essential for the most advanced technologies. So far, many studies of the production of high density carbon artefacts have been reported. The processes for preparing carbon artefacts are typically classified into two categories. Procedures in the first category use filler coke and binder pitch as starting materials. The process consists of moulding the mixture by CIP (Cold Isostatic Press), carbonization (baking) followed by impregnations, and graphitization into the final product. The carbonization and impregnation are often repeated for densification. The second process consists of preparation of self-adhesive carbonaceous grains, moulding them without any additional binding substances, carbonization, and graphitization. No necessity of the binder and the impregnation steps is a characteristic of this process. Carbon in small quantities is added to iron, 'Steel' is obtained. Since the influence of carbon on mechanical properties of iron is much larger than other alloying elements. The atomic diameter of carbon is less than the interstices between iron atoms and the carbon goes into solid solution of iron. As carbon dissolves in the interstices, it distorts the original crystal lattice of iron. This mechanical distortion of crystal lattice interferes with the external applied strain to the crystal lattice, by mechanically blocking the dislocation of the crystal lattices.

Conventionally, carbon blocks are fabricated from the filler of coke and the binder of pitch via a series of complex processing steps involving mixing of raw materials, molding, many times of dipping and pyrolysis, and graphitization at elevated temperatures. The mesophase pitches exhibited high coke yield, low softening point, and high fluidity.

Mesophase pitches are considered to be good starting material for many industrial and advanced carbon products, such as carbon fibres, needle coke, graphite electrodes, C-C composites, fine-grained sintered carbons, Li-ion battery anodes, mesocarbon microbeads, carbon foam and plasma-facing components for fusion devices etc. Carbonaceous mesophase is a self-sintering precursor, which is able to produce polygranular carbons and graphites. The sinterability of mesophase derives from its intrinsic thermoplastic properties that allow mesophase to be moulded and compacted, giving rise to highdensity and high-strength artefacts. Several researchers studied the self-sinterability of mesocarbon microbeads (MCMB) for preparation of high-density isotropic carbon [1,2]. Yongzhong Song et al. [3] prepared graphite seal materials from mesocarbon microbeads. Silicon

${ }^{*}$ Corresponding author: Singh Arora SP, Scientist/Engineer-SD, Indian Space Research Organisation (ISRO), IPRC (M), Govt. of India, Tamil Nadu, India, Tel: 8022172465; E-mail: mikkiiarora@rediffmail.com

Received August 15, 2016; Accepted January 02, 2017; Published January 12, 2017

Citation: Deepak Kumar G, Singh Arora SP (2017) Evaluation of Physico-Chemical, Thermal and Mechanical Properties of Sintered Graphite and Mesophase Formulations. J Material Sci Eng 6: 314. doi: 10.4172/2169-0022.1000314

Copyright: () 2017 Deepak Kumar G, et al. This is an open-access article distributed under the terms of the Creative Commons Attribution License, which permits unrestricted use, distribution, and reproduction in any medium, provided the original author and source are credited. 
carbide $(\mathrm{SiC})$ is one of the most promising structural materials due to its superior thermomechanical properties, such as high chemical and thermal stability, good chemical inertness, high thermal conductivity, high hardness, low density, and low coefficient of thermal expansion. Silicon carbide is a compound of silicon and carbon with chemical formula SiC. It occurs in nature as mineral moissanite. Because of the rarity of natural moissanite, most silicon carbides are synthetic. $\mathrm{SiC}$ is part of a family of materials that exhibit a one-dimensional polymorphism called polytypism. Thanks to its structure, an almost infinite number of $\mathrm{SiC}$ polytypes are possible, and more than 200 have already been discovered. With the introduction of nanotechnology and manipulation at nanoscale, new opportunities have been opened. Onedimensional (1D) nanostructures such as nanowires or nanotubes have gained much interest in fundamental research as well as tremendous potential applications. Among many materials, SiC-based 1D nanostructure has very interesting physical, chemical, and electronic properties. $\mathrm{SiC}$ is a wide band gap semiconductor, from $2.2 \mathrm{eV}$ to $3.4 \mathrm{eV}$ depending on the polytype with a high electronic mobility and thermal conductivity. One of the other merits of $\mathrm{SiC}$ is that it can be used at high temperatures because its noncongruent melting point reaches $3100^{\circ} \mathrm{K}$ and its maximal operating temperature is considered to be $1200^{\circ} \mathrm{K}$. Moreover, $\mathrm{SiC}$ is resistant to corrosive environments because of its chemical inertness. These facts justify the high application of SiC in a wide range of areas, such as electronics, heating elements, and structural materials. More particularly, $\mathrm{SiC}$ nanoparticles have been introduced in CMCs to enhance the mechanical and thermal properties of ceramic materials such as $\mathrm{Al}_{2} \mathrm{O}_{3}$ and mullite. However, the thermo plasticity of the mesophase is usually too high, and consequently, after being moulded into rigid pieces, the mesophase deforms and distorts in subsequent carbonizations. It is necessary, therefore, to modify thermoplastic properties of the mesophase before sintering. A process widely used to reduce plasticity in carbon precursors is oxidative stabilization at moderate temperatures. Isao Mochida et al. [4] prepared the self-adhesive carbonaceous grains through the oxidation of the mesophase pitches synthesized from naphthalene. Fanjul et al. [5] prepared high-density polygranular carbons and graphites from carbonaceous mesophase, to study the changes in the chemical composition of the mesophase obtained from a coal-tar pitch and a naphthalene-based pitch at different stages of oxidative stabilization with air $\left(200-300^{\circ} \mathrm{C}\right)$, and the carbonization behaviour of samples stabilized at different temperatures below $1000^{\circ} \mathrm{C}$.

\section{Related Work}

In view of the above the objective of this analysis is focused on the development of high density graphite material from a mixture of graphite powder and mesophase pitch with the target properties as depicted in Table 1 for possible application as pump seals at cryogenic temperatures. Attempts will also be made to design and fabricate a

\begin{tabular}{|l|l|l|}
\hline S.no & Properties & Required values \\
\hline 1 & Density $(\mathrm{g} / \mathrm{cc})$ & 1.75 to 2.10 \\
\hline 2 & Surface conditions & $\begin{array}{l}\text { The surface should be free from } \\
\text { cracks, delaminations, blisters etc. }\end{array}$ \\
\hline 3 & Presence of anisotropic pyrographite & Minimum \\
\hline 4 & Presence of particulates & Nil \\
\hline 5 & Thermal conductivity $(\mathrm{W} / \mathrm{m} / \mathrm{k})$ & 30 to 50 \\
\hline 6 & $\begin{array}{l}\text { Linear coefficient of thermal } \\
\text { expansion }\left(\mathrm{K}^{-1}\right)\end{array}$ & $(6.5 \text { to } 8.5)^{\star} 10^{-6}$ \\
\hline 7 & Electrical resistivity $(\mathrm{Ohm} \mathrm{m})$ & $(3.08 \text { to } 4.36)^{\star} 10^{-5}$ \\
\hline 8 & compressive strength $(\mathrm{MPa})$ & 190 to 360 \\
\hline
\end{tabular}

Table 1: Target properties of seal material. die for shaping of the formulation into a seal to establish the shaping capability.Our system aims at the automatic detection of text.

Selection and characterisation of starting raw materials, milling and mixing followed by the physico-chemical, morphological and thermal properties evaluation

Commercially available graphite powder and mesophase pitch (the precursor for graphite) binder were ball milled. The particle morphology before and after milling was observed under Scanning Electron Microscope (Hitachi, Tokyo, Japan). The particle size was measured using Laser Diffraction Technique (Malvern, UK). The mixtures have shown the flaky and irregular morphology (Figure 1). The graphite and mesophase pitch shows crystalline and amorphous nature, respectively. Graphite-mesophase pitch mixtures with graphite to mesophase pitch ratio of 90:10 (designated as GM-I), 80:20 (designated as GM-II) and 70:30 (designated as GM-III) were prepared. The mixtures contain particle with sizes in the range of $4-15 \mu \mathrm{m}[6,7]$.

\section{Compaction of formulations by die, compaction experiments,} characterization and heat treatments of the compacts

Compaction behavior was elucidated by feeding $\sim 0.5 \mathrm{~g}$ of the mix individually into the compaction die with $10 \mathrm{~mm}$ diameter (Figure 2) and compacted using Universal Testing Machine (Instron, 4483) (Figure 3) [8]. Load-displacement values were recorded using a computer interface. In all the three mixes while carrying out the compaction process initially the powder was compacted to an initial pressure of $5 \mathrm{MPa}$ the displacement values of the top punch were recorded. All mixes were compacted at a maximum pressure of 550 $\mathrm{MPa}$ with definite increment of pressure and for every pre-set value of these pressures the displacements of the top punch from the initial position are recorded $[9,10]$.

\section{Experimental Results}

Plot of variation of the thickness of the compacts for Mix-I, Mix-II and Mix-III plotted by the method of periodic loading and unloading under compaction process are shown in Figure 4 . The curve depicted in Figure 4 demonstrates the compaction process and associated elastic stresses on the compact. When the sample is under compaction pressure the strain energy is stored in the pellets, on release of compaction stresses the pellet expands in axial direction while the pellet is still constrained within the die walls.

Density of the ejected compacts for each mix was also determined by measurement of weight and dimensions. Mix-I with the maximum graphite content (90\%) exhibited a spring back of $12 \%$, however, attained a maximum density of $89 \%$ of the theoretical density $(2.205 \mathrm{~g} /$ cc). In case of Mix-II and Mix-III exhibited densities of 86 and $85 \%$ of theoretical density, where the theoretical densities are 2.150 and 2.095 $\mathrm{g} / \mathrm{cc}$ respectively. As the mesophase pitch content in the mix increases from $10 \%$ to $30 \%$ it facilitates compaction by better bonding and hence reduces the spring back. It is evident from Table 2 that after normalizing
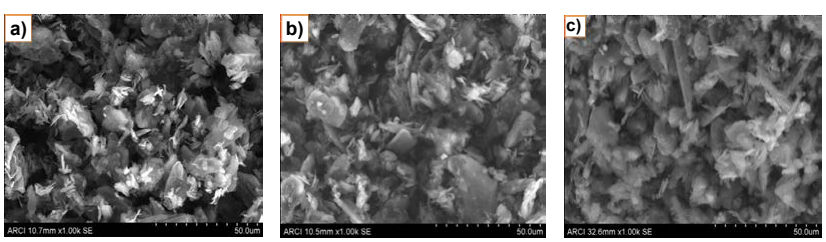

Figure 1: Morphology of (a-c) Mix-I, Mix-II and Mix-III. 
Citation: Deepak Kumar G, Singh Arora SP (2017) Evaluation of Physico-Chemical, Thermal and Mechanical Properties of Sintered Graphite and Mesophase Formulations. J Material Sci Eng 6: 314. doi: 10.4172/2169-0022.1000314
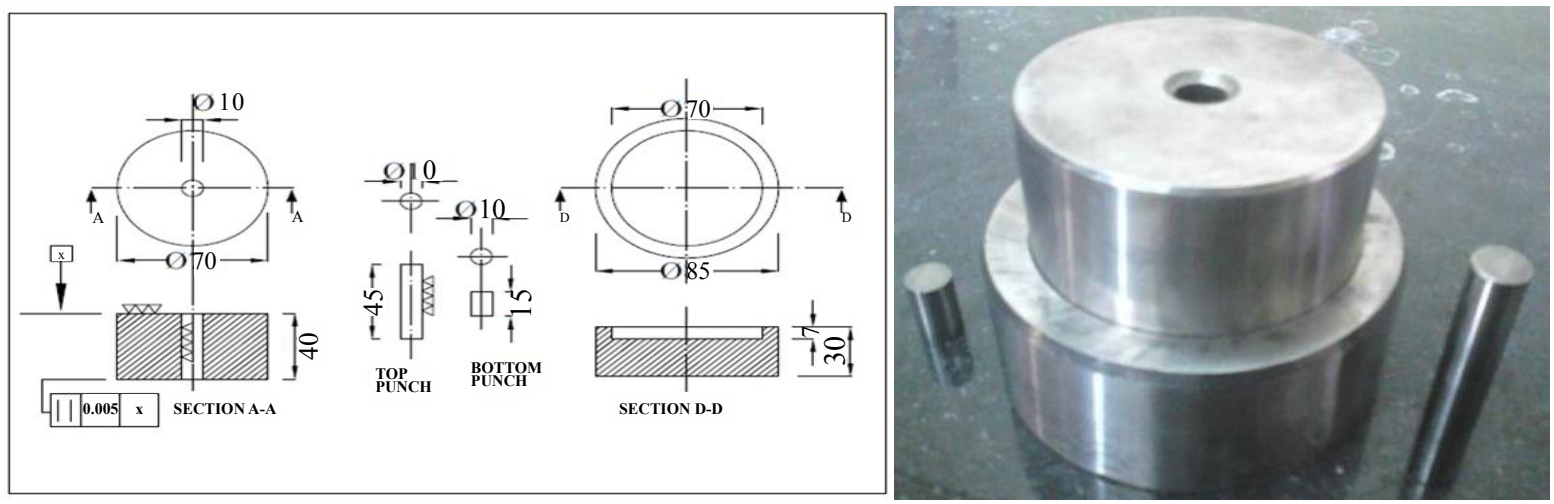

Figure 2: Shows the Engineering drawing and photograph of the Compaction Die.

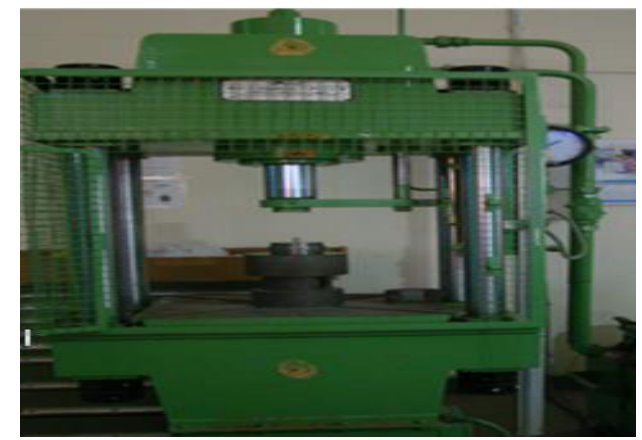

Figure 3: Shows the hydraulic press and compaction die along with bottom and top punches.
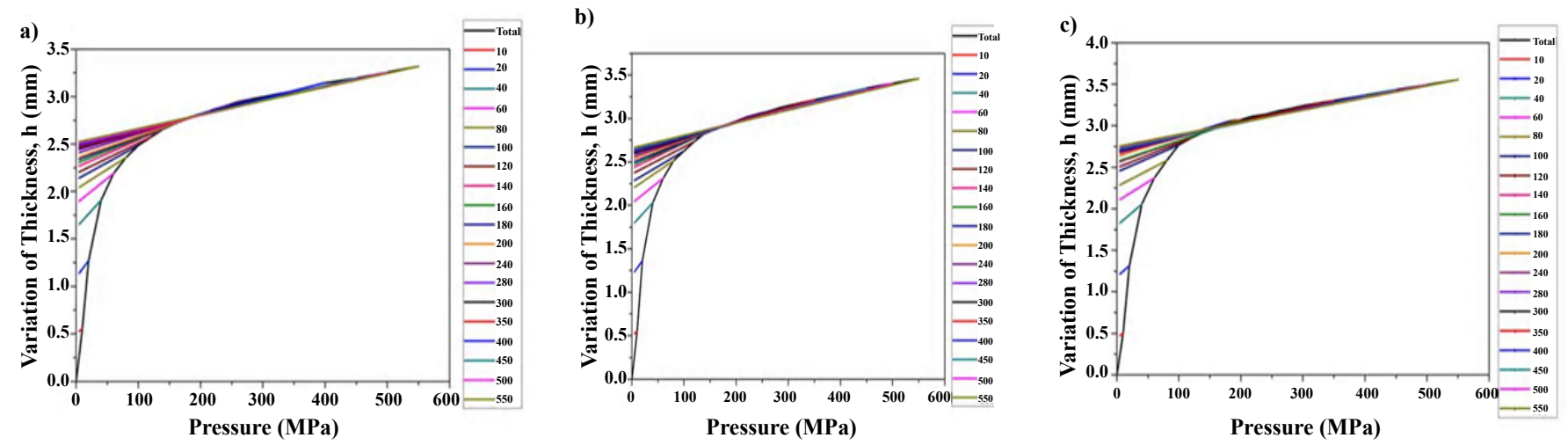

Figure 4: Variation of the thickness $\Delta \mathrm{h}$ of a pressing graphite and mesophase pitch powder in the compaction process plotted by the method of periodic loading and unloading of the pressure (a) Mix-I (b) Mix-II and Mix-III powders.

by the rule of mixtures, though spring back is less, densities achieved for Mix-II and Mix-III are less which is not desirable for compaction processing. Though spring back is significant by $12 \%$ in case of Mix-I there is no introduction of any lamination defects.

It is also interesting to note that though spring back is significant the samples are defect free as is evidenced by the high density value. The study reveals that a mix of graphite and mesophase pitch in the ratio of 90: 10 is the best choice for the compaction processing.

All the cold compacted samples were subjected to carbonization at $1200^{\circ} \mathrm{C}$ in argon atmosphere. We have observed development of cracks throughout all the samples (Figure 5) due to the escape of organics even with very low heating rates and also due to the too high thermoplasticity of mesophase pitch. Therefore, it is necessary to modify the thermoplastic properties of mesophase pitch before sintering to eliminate the cracks formation. A process widely used to reduce plasticity in carbon precursors is oxidative stabilization at moderate temperatures. The compacted samples of all the three mixtures were heat treated at a temperature of $250^{\circ} \mathrm{C}$ for oxidative stabilization purpose. The compact obtained from GM-I mixture produced the sample without any crack after the carbonization (Figure 6). However, the sample densities observed is in the range of 1.65 to $1.7 \mathrm{~g} / \mathrm{cc}$. Alternatively, the 
Citation: Deepak Kumar G, Singh Arora SP (2017) Evaluation of Physico-Chemical, Thermal and Mechanical Properties of Sintered Graphite and Mesophase Formulations. J Material Sci Eng 6: 314. doi: 10.4172/2169-0022.1000314

Page 4 of 5

mixtures were subjected to oxidative stabilization before compaction. Subsequent carbonization results in crack free samples only for GM-I.

Exploration of advanced compaction techniques such as Hot Pressing as shaping options followed by graphitization experiments at high temperatures $>2000^{\circ} \mathrm{C}$

In view of this GM-I mixture was subjected to hot pressing at temperature of $1200^{\circ} \mathrm{C}$ and at pressures of 12,15 and $18 \mathrm{MPa}$. The samples exhibited a maximum density of $1.7 \mathrm{~g} / \mathrm{cc}$ under hot compaction under $15 \mathrm{MPa}$, however, resulted in lamination cracks beyond 15 $\mathrm{MPa}$. The low density of $1.7 \mathrm{~g} / \mathrm{cc}$ compared to cold compaction even after simultaneous application of temperature and pressure can be attributed to the low pressure involved in hot pressing due to the strength limitation of the graphite dies used. The hot pressed samples

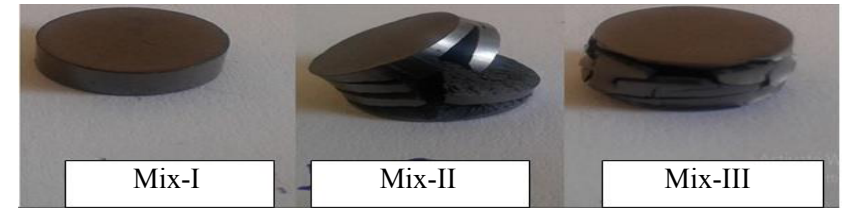

Figure 5: Shows Sample images of Mix-I, II, III compositions after carbonization

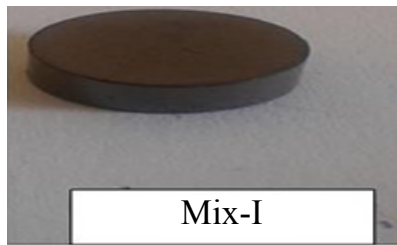

Figure 6: Shows the sample image of Mix-I with no Crack after carbonization. were graphitized at $2500^{\circ} \mathrm{C}$ in a resistive heating graphite furnace. Unlike carbonized samples, the graphitized samples have shown highly crystalline graphite phase with an improved thermal conductivity of $48.26 \mathrm{~W} / \mathrm{m} / \mathrm{K}$ complementing the density and XRD results. A significant decrease in the electrical resistivity has also been observed which can be attributed to the above cited reasons. A thermal expansion value has exhibited only marginal increase. However, there is a significant increase in compressive strength achieving a maximum of $60 \mathrm{MPa}$ and a low coefficient of friction 0.08 which are in good agreement with the improved density values. The properties of hot-pressed and graphitized samples are listed in Table 3.

Fabrication of the die followed by compaction experiments to establish the shaping suitability of the raw mix into seals

To study the adaptability for shaping a die has been fabricated and GM-I was compacted at a pressure of $500 \mathrm{MPa}$ into the circular ring for seal application by die (Figure 7). The photograph of the fabricated rings is shown in Figure 7.

\section{Conclusion}

Comparative evaluation of compaction behavior of graphitemesophase pitch mixtures of graphite to mesophase pitch ratio of 90:10 (GM-I), 80:20 (GM-II) and 70:30 (GM-III) prepared by planetary ball

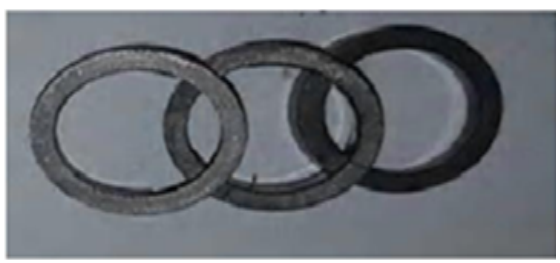

Figure 7: Photograph of the circular rings fabricated by compaction of GM-1.

\begin{tabular}{|l|c|c|c|}
\hline Sample & Compaction pressure (Mpa) & Green density (g/cc) & Theoretical density *(g/cc) \\
\hline Mix-I & 550 & 1.984 & 2.205 \\
\hline Mix-II & 550 & 1.8525 & 2.105 \\
\hline Mix-III & 550 & 1.784 & 89 \\
\hline
\end{tabular}

*Estimated based on the rule of mixtures (Graphite $-2.26(\mathrm{~g} / \mathrm{cc})$ and Mesophase pitch-1.71 (g/cc))

Table 2: Green densities of sample.

\begin{tabular}{|c|l|c|c|}
\hline S. no & Properties & Hot pressed (carbonised sample) & Graphitized sample \\
\hline 1 & Density $(\mathrm{g} / \mathrm{cc})$ & 1.7 & 1.78 \\
\hline 2 & Surface conditions & Free from cracks, delaminations, and blisters & Free from cracks, delaminations, and blisters \\
\hline 3 & Presence of particulates & 12.23 & Nil \\
\hline 4 & Thermal conductivity $(\mathrm{W} / \mathrm{m} / \mathrm{k})$ & $12^{*} 10^{-6}$ & 48.26 \\
\hline 5 & Linear cofficient of thermal expansion $\left(\mathrm{k}^{-1}\right)$ & $5.475^{\star} 10^{-5}$ & $13.4^{\star} 10^{-6}$ \\
\hline 6 & Electrical resistivity $($ Ohm $\mathrm{m})$ & 49 & $3.21^{\star} 10^{-5}$ \\
\hline 7 & Compressive strength & 60 \\
\hline
\end{tabular}

Table 3: Properties of hot pressed and graphitized samples.

\begin{tabular}{|c|l|c|c|}
\hline S. no & Properties & Values targeted & 1.75 to 2.10 \\
\hline 1 & Density $(\mathrm{g} / \mathrm{cc})$ & Surface should be free from cracks, delaminations, blisters etc. & Free from cracks, delaminations and blisters \\
\hline 2 & Surface conditions & Minimum \\
\hline 3 & Presence of anisotropic pyrographite & Nil \\
\hline 4 & Presence of particulates & 30 to 50 \\
\hline 5 & Thermal conductivity $(\mathrm{W} / \mathrm{m} / \mathrm{k})$ & $(6.5 \text { to } 8.5)^{*} 10^{-6}$ \\
\hline 6 & Linear coefficient of thermal expansion $\left(\mathrm{k}^{-1}\right)$ & $(3.08 \text { to } 4.36)^{*} 10^{-5}$ & 1.78 \\
\hline 7 & Electrical resistivity $($ Ohm $\mathrm{m})$ & 190 to 360 & $13.4^{*} 10^{-6}$ \\
\hline 8 & Compressive strength & $3.21^{*} 10^{-5}$ \\
\hline
\end{tabular}

Table 4: Properties of Achieved by the graphitized sample. 
Citation: Deepak Kumar G, Singh Arora SP (2017) Evaluation of Physico-Chemical, Thermal and Mechanical Properties of Sintered Graphite and Mesophase Formulations. J Material Sci Eng 6: 314. doi: 10.4172/2169-0022.1000314

Page 5 of 5

milling were studied by uni-axial loading and unloading technique using a universal testing machine. Spring back on release of compaction stresses are found to be higher in case of GM-I as evident from the plots exhibiting elastic interaction in comparison to GM-II and GMIII formulations. Higher normalized density values are achieved in case of GM-I in spite of significant spring back can be attributed to the optimum concentration of mesophase pitch in the formulation.

Though cold compaction at a high pressure of $500 \mathrm{MPa}$ has resulted in higher density values, exhibited cracks while heat treatment. Simultaneous application of temperature and pressure by hot pressing has yield carbonized samples with relatively low densities without cracks. Graphitization of hot pressed compact at $2500^{\circ} \mathrm{C}$ has shown a maximum density of $1.78 \mathrm{~g} / \mathrm{cc}$ and enhancement of thermal, mechanical, wear and electrical properties as revealed by Table 3 .

Properties of high density graphite proposed to develop from graphite and mesophase pitch in the study and the properties achieved are summarised as below. Table 4 clearly indicates that the properties such as density, electrical resistivity, thermal conductivity could be achieved or closes to the required values.

\section{Future Directions}

Minimisation of presence of anisotropic pyrographite could not be attempted in the study and the variation in the properties can be attributed to the random orientation of graphite crystals. Future work can be focused on addressing this issue by carrying out hot isostatic pressing.

\section{References}

1. Gao Y, Song H (2003) Self-sinterability of mesocarbon microbeads (MCMB) for preparation of high-density isotropic carbon. Journal of Materials Science 38: 2209-2213.

2. Yuan G, Li X (2012) Graphite blocks with preferred orientation and high thermal conductivity. Carbon 50: 175-182.

3. Song Y, Zhai G (2004) Carbon/graphite seal materials prepared from mesocarbon microbeads. Carbon 42: 1427-1433.

4. Mochida I (1994) Self-adhesive carbon grains oxidatively prepared from naphthalene derived mesophase pitch for mould of high density. Carbon 32: 961-969.

5. Fanjul F, Granda M, Santamar R, Menéndez R (2003) Pyrolysis behaviour of stabilized self-sintering mesophase. Carbon 41: 413-422.

6. Zhao Y, Liu Z (2013) Microstructure and thermal/mechanical properties of short carbon fiber-reinforced natural graphite flake composites with mesophase pitch as the binder. Carbon 53: 313-320.

7. Zhong B, Zhao GL (2014) Binding natural graphite with mesophase pitch: A promising route to future carbon blocks. Materials Science \& Engineering A 610: $250-257$

8. Fang MD, Tseng WL, Jow JJ (2012) Improving the self-sintering of mesocarbonmicrobeads for the manufacture of high performance graphite-parts. Carbon 50: $906-913$.

9. Mochida I, Korai Y (2000) Chemistry of synthesis, structure, preparation and application of aromatic-derived mesophase pitch. Carbon 38: 305.

10. Liub Z, Guo Q, Shi J, Zhai G, Liu L (2008) Graphite blocks with high thermal conductivity derived from natural graphite flake. Carbon 46: 414-421. 